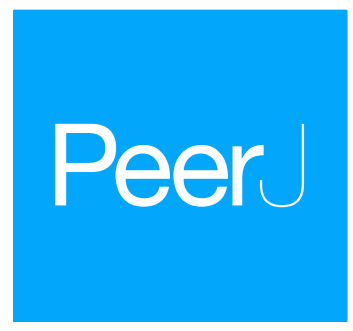

\title{
A comparison of least squares regression and geographically weighted regression modeling of West Nile virus risk based on environmental parameters
}

\author{
Abhishek K. Kala ${ }^{1}$, Chetan Tiwari ${ }^{2}$, Armin R. Mikler ${ }^{3}$ and Samuel F. Atkinson ${ }^{1}$ \\ Advanced Environmental Research Institute and Department of Biological Sciences, University of \\ North Texas, Denton, TX, United States \\ ${ }^{2}$ Advanced Environmental Research Institute and Department of Geography and the Environment, University \\ of North Texas, Denton, TX, United States \\ Advanced Environmental Research Institute and Department of Computer Science and Engineering, \\ University of North Texas, Denton, TX, United States
}

\section{ABSTRACT}

Background. The primary aim of the study reported here was to determine the effectiveness of utilizing local spatial variations in environmental data to uncover the statistical relationships between West Nile Virus (WNV) risk and environmental factors. Because least squares regression methods do not account for spatial autocorrelation and nonstationarity of the type of spatial data analyzed for studies that explore the relationship between WNV and environmental determinants, we hypothesized that a geographically weighted regression model would help us better understand how environmental factors are related to WNV risk patterns without the confounding effects of spatial nonstationarity.

Methods. We examined commonly mapped environmental factors using both ordinary least squares regression (LSR) and geographically weighted regression (GWR). Both types of models were applied to examine the relationship between WNV-infected dead bird counts and various environmental factors for those locations. The goal was to determine which approach yielded a better predictive model.

Submitted 8 September 2016

Accepted 7 February 2017

Published 28 March 2017

\section{Corresponding author}

Samuel F. Atkinson,

atkinson@unt.edu

Academic editor

Andrew Noymer

Additional Information and Declarations can be found on page 13

DOI 10.7717/peerj.3070

Copyright

2017 Kala et al.

Distributed under

Creative Commons CC-BY 4.0
Results. LSR efforts lead to identifying three environmental variables that were statistically significantly related to WNV infected dead birds (adjusted $R^{2}=0.61$ ): stream density, road density, and land surface temperature. GWR efforts increased the explanatory value of these three environmental variables with better spatial precision (adjusted $R^{2}=0.71$ ).

Conclusions. The spatial granularity resulting from the geographically weighted approach provides a better understanding of how environmental spatial heterogeneity is related to WNV risk as implied by WNV infected dead birds, which should allow improved planning of public health management strategies.

OPEN ACCESS

Subjects Biogeography, Environmental Sciences, Epidemiology, Infectious Diseases, Public Health

Keywords Emerging infectious diseases, Avian impacts, West Nile virus, Spatial modeling, Geographic information systems (GIS), Model comparison 


\section{INTRODUCTION}

West Nile Virus (WNV) is a vector-borne disease that was first detected in the United States in 1999 (Nash et al., 2001). Within a few years the virus had spread across the North American continent (Hayes et al., 2005). WNV has had important environmental and human impacts, including a decline in numerous bird species $(C D C)$ and increased morbidity and mortality among humans. This has also resulted in increased economic burdens due to initial acute health care needs of infected individuals and subsequent long-term costs associates with infection, estimated at approximately $\$ 56$ million per year between 1999 and 2012 (Barrett, 2014). Because that study indicated how difficult predicting and planning for WNV outbreaks was, we became interested in developing a spatially explicit model using environmental factors in an attempt to improve WNV risk predictions.

There are two important considerations that should typically be examined when developing spatially explicit environmental disease risk models (Miller, 2012). The first should be an examination of potential spatial autocorrelation (the degree to which a set of spatial features and their associated data values tend to be clustered together in space). This involves accounting for whether environmental factors and the corresponding disease rates in geographically proximate areas are more or less clustered together than they are in geographically distant areas. Second, data non-stationarity (changing means, variances and covariances in data across space) should be investigated and controlled when necessary (Fotheringham, 2009a; Miller, 2012). Geographically weighted regression (GWR) can be used for these two considerations and can often produce improved models that enable better spatial inference and prediction. Recent studies have applied GWR modeling to drug-resistant tuberculosis versus risk factors (Liu et al., 2011); environmental factors versus typhoid fever (Dewan et al., 2013); local climate and population distribution versus hand, foot, and mouth disease (Hu et al., 2012); and environmental factors and tick-borne disease (Atkinson et al., 2012; Atkinson et al., 2014; Wimberly, Baer \& Yabsley, 2008; Wimberly et al., 2008), all showing that predictor variables varied spatially across large geographic regions, implying that the results for such studies may be improved using GWR.

The spatially explicit model that is discussed in this paper uses GWR to account for spatial heterogeneity for two reasons: (a) WNV disease risk observed across space may be related to similar environmental variables that increase vector habitat suitability and (b) environmental variables that influence WNV risk are not typically uniformly distributed across geographic space. Although many epidemiological models of WNV risk have been developed, it appears that there has been little research to explicitly examine techniques that account for spatial heterogeneity. Most models assume that the impact of various environmental factors are constant across the study region, which is unrealistic as larger areas display substantial variations in distribution of environmental, socio-economic, and demographic conditions (Goovaerts, 2008).

Due to the unavailability of reliable and complete data, developing models of WNV risk pose additional challenges. Human case data is lacking due to issues of under-reporting and limited surveillance. Our alternative strategy was to assess WNV infected dead bird counts as a surrogate measure of human risk because "infection rates" in dead birds can 
be more precise because of the genetic markers tested in dead birds may be more reliable than case data and/or surveillance data. Additionally, others have also used mosquito habitat suitability as a surrogate for estimating WNV risk for human infection (Cooke, Grala \& Wallis, 2006). For our study, we followed a similar approach and used a model of mosquito habitat suitability condition as a predictor of the spatial distributions of infected birds, which in turn can be used to estimate WNV disease risk among human populations. Further, because the environmental variables considered in this study are known to vary across space, we account for spatial autocorrelation and non-stationarity using GWR following the approach of (DeGroote et al., 2008) in order to improve the predictability of a model.

\section{WNV transmission and risk factors}

The WNV transmission cycle was an important component of our modeling efforts. The first step in the WNV transmission cycle primarily occurs when a competent female mosquito vector bites an infected bird reservoir host, which in turn results in the virus being transmitted to the mosquito (Blair, 2009). This occurs when the female mosquito is seeking a blood meal to obtain nutrients necessary for egg development. After taking an infectious blood meal, a mosquito may pick up a permanent infection. The infected mosquito now has the potential to transmit the virus to another bird or animal when it feeds again. Once infected, birds may fly to other locations where the virus can be transmitted to susceptible mosquitoes. Subsequently, the disease may be transmitted by infected mosquitoes to humans or other mammals that act as incidental hosts. Dead birds found to be infected with WNV are often the primary indicators for presence of the disease in a geographic region and have proven to be useful for disease prediction modeling and identifying areas for human infection risk (Cooke, Grala \& Wallis, 2006; Ruiz et al., 2004; Valiakos et al., 2014). This relationship allows an assumption of a positive correlation between infected dead birds and WNV risk. Since the New York outbreak in 1999, WNV has been recovered from 26 mosquito species in North America, including Culex. pipiens, Culex. salinarius, Culex. restuans, Ochlerotatus canadensis, Oc. japonicus, Aedes vexans, and Culiseta melanura (CDC, 2000; Control and Prevention, 2001). Results from a study (Goddard et al., 2002) assesses the vector competence of California mosquitoes. The results indicate that mosquitoes in the genus Culex $(C x$.$) are the principal hosts of WNV$ in California. The study also analyzed that on the basis of vector competence and hostfeeding patterns, $C x$. tarsalis may be the principal vector in rural agricultural ecosystems; and $C x$. pipiens complex and $C x$. stigmatosoma as important vectors in urban settings.

Vector and pathogen reservoirs overlap when certain environmental conditions are present (Rochlin et al., 2011). Table 1 provides an overview of the environmental conditions that are associated with WNV transmission, which were utilized for our research. These include characteristics of a place such as the mosquito species habitat: climatic conditions, topography and land use/land cover classes such as vegetation, water, and urbanized areas. Spectral indices acquired from satellite imagery provide information about environmental characteristics like temperature, vegetation cover, and moisture (Rodgers \& Mather, 2006). Liu \& Weng (2012) in a study on WNV risk in southern California found that one of the 
Table 1 Environmental conditions related to WNV transmission risk.

\begin{tabular}{|c|c|c|}
\hline Factors studied & Relation to WNV & References \\
\hline Streams & Sites for breeding and resting & $\begin{array}{l}\text { Cooke, Grala \& Wallis (2006), Curtis et al. (2014) and } \\
\text { Schurich et al. (2014) }\end{array}$ \\
\hline Temperature & $\begin{array}{l}\text { Increases growth rate of vector, decreases egg development } \\
\text { cycle and shortens extrinsic incubation period of vector }\end{array}$ & $\begin{array}{l}\text { DeGroote, Sugumaran \& Ecker (2014), Kuehn (2012), } \\
\text { Srivastava et al. (2001) and Wimberly et al. (2008) }\end{array}$ \\
\hline Surface slope & Water stagnation creating mosquito breeding ground & $\begin{array}{l}\text { Cooke, Grala \& Wallis (2006), Ozdenerol, Bialkowska-Jelinska \& } \\
\text { Taff (2008), Schurich et al. (2014) and Srivastava et al. (2001) }\end{array}$ \\
\hline Cultivated land & $\begin{array}{l}\text { Linkage between habitat used and human-commensal } \\
\text { nature of WNV mosquito vectors }\end{array}$ & Kilpatrick (2011) \\
\hline Developed land & $\begin{array}{l}\text { Linkage between habitat used and human-commensal } \\
\text { nature of WNV mosquito vectors; warmer microclimates }\end{array}$ & Kilpatrick (2011) \\
\hline Roads & Sites for breeding and resting along roadsides & Cooke, Grala \& Wallis (2006) \\
\hline Vegetation & Sites for breeding and resting. & $\begin{array}{l}\text { Brownstein et al. (2002), Cooke, Grala \& Wallis (2006), } \\
\text { DeGroote et al. (2008), Ruiz et al. (2004), Schurich et al. (2014) } \\
\text { and Srivastava et al. (2001) }\end{array}$ \\
\hline Evapotranspiration & $\begin{array}{l}\text { Related to the amount of moisture that is related to } \\
\text { mosquito abundance }\end{array}$ & Liu \& Weng (2012) and Trawinski \& Mackay (2008) \\
\hline
\end{tabular}

main factors contributing to the WNV propagation included land surface temperature. They related higher temperature to viral replication in mosquitoes for WNV to be disseminated throughout the year. The results also show that areas with lower elevations tended to be more susceptible to WNV invasion as mosquito population propagates in the plain habitats with warmer temperatures compared to areas with higher elevation that have lower temperatures.

\section{Statistical Considerations}

Miller (2012) suggests that a 'global' model is the one that assumes that the parameters (commonly mean and variance) of some process are constant across geographic space (commonly mean and variance), typically referred to as the spatial stationarity of a process. Miller suggests that in the case these parameters vary across geographic space (spatial heterogeneity), then such models may lead to inaccurate predictions and subsequent problems for decision-making. In an ecological context, spatial heterogeneity usually results from the interaction of various environmental processes that operate at different scales (Legendre, 1993). Fotheringham (2009b) used local statistics for linking the concepts of spatial autocorrelation and heterogeneity that are deemed important when developing spatial models. Local statistics disaggregate a global mean value into locally computed values for each spatial unit. It is based on a conceptualization of Tobler's first law in Geography (Tobler, 1970) that specifies that "everything is related to everything else, but near things are more related than distant things." Spatial autocorrelation is a commonly used measure of the degree of spatial heterogeneity.

GWR is a local regression method that can be used for diagnosing spatial heterogeneity between dependent and explanatory variables over space (Fotheringham, Brunsdon \& Charlton, 2003). It is performed within local windows centered on the nodes of a regular grid. Each observation within the local window is weighted based on its proximity to the 
center of that window. This approach has several advantages: it avoids abrupt changes in the local statistics computed for adjacent windows, helps visualize spatial variability within the geographic entity, and allows analysis of regionally aggregated data (Goovaerts, 2008). A model's predictive ability, particularly in ecological modeling, is influenced not only by the strength of relationships between the species and its environment, but whether the model recognizes if the relationships are operating at multiple spatial scales. GWR provides a framework for exploring scale-dependent effects. It tests the effect on a model's predictive ability by systematically increasing the size of the local window (Miller, 2012).

GWR can be used for mapping the spatial distribution of a model's coefficient values in order to identify potential missing variables or to suggest other underlying factors associated with the observed non-stationarity (Miller, 2012). GWR is also useful for exploratory data analysis and visualization; for example Kupfer \& Farris (2007) used a 'leave-one-out' (jackknifing) methodology to compare residuals from GWR and ordinary least squares regression. They found that GWR often had more accurate predictions for sites that were difficult to predict (where both models had overall higher residuals), which is why we used a GWR framework for explicitly modeling the spatial relationships between WNV and its environmental risk factors.

\section{MATERIALS \& METHODS}

\section{Study area}

The model was built for the state of California, which was the national epicenter of WNV activity in 2004 and 2005 (Jean et al., 2007). WNV was first detected there in July 2003 (Reisen et al., 2004). It is the third largest state by area in the United States and is made up of 58 counties. California has the largest population in the US, but it is unevenly distributed across the state. The state also has a variable landscape with a large valley in the middle, bounded by mountain ranges.

\section{Environmental factors and data sources}

Our model utilized various environmental factors (Table 1) that have been suggested as descriptive in local WNV risk distribution: surface slope, density of roads, density of streams, monthly mean temperature, monthly mean evapotranspiration, and land cover classes like vegetation, developed land, cultivated land, and open surface water. All environmental parameters except roads and streams (Table 2) were acquired in grid format and resampled to $120 \mathrm{~m}$ resolution as suggested by Cooke, Grala \& Wallis (2006). Data resampling was done using the resampling tool available in ArcGIS software. The modeling method utilized in this study was based on analyzing data in raster format, and therefore road and stream vector data were converted to raster format using the 'Kernel Density Estimation' tool in ArcGIS to create road density and stream density grid files. The tool assumes a Gaussian distribution and thus assigns more importance towards the center of kernel in comparison to the features that are further apart.

Various dynamic environmental data including Normalized Difference Vegetation Index (NDVI), Land Surface Temperature (LST), and Evapotranspiration (ET) were downloaded from the Moderate Resolution Imaging Spectroradiometer (MODIS) toolbox incorporated 
Table 2 Data sources.

\begin{tabular}{lll} 
Data & Spatial resolution & Source \\
\hline Elevation & $10 \mathrm{~m}$ & National Elevation Dataset (NED) \\
LST & $1 \mathrm{~km}$ & MODIS aboard the Terra and Aqua satellites \\
NDVI & $250 \mathrm{~m}$ & MODIS aboard the Terra and Aqua satellites \\
Evapotranspiration (ET) & $1 \mathrm{~km}$ & MODIS aboard the Terra and Aqua satellites \\
Streams & Available in vector format & US bureau of reclamation \\
Roads & Available in vector format & US Census bureau \\
Cultivated land & $30 \mathrm{~m}$ & National Land Cover Database \\
Developed land & $30 \mathrm{~m}$ & National Land Cover Database \\
WNV infected dead birds count & County scale & USGS National wildlife health center \\
WNV human incidence cases & County scale & USGS National wildlife health center \\
Human population & County scale & US Census bureau
\end{tabular}

in ArcGIS ${ }^{\circledR}$. The Land Surface Temperature tool accesses MOD11-A1, the daily averaged LST product. The MOD11 product uses the algorithm which is optimally used to separate ranges of atmospheric column water vapor and lower boundary air surface temperatures into tractable sub-ranges. The NDVI is calculated according to the formula NDVI $=(\mathrm{NIR}$ - VIS)/(NIR + VIS) where NIR is the near-infrared radiance and VIS is observed radiation in the visible spectrum. NDVI data is available from either satellite with MODIS (Aqua or Terra) as a monthly average. The time lag between the hatching of a mosquito egg to an adult mosquito taking blood meals and becoming infected with WNV to the subsequent infection of a human and the appearance of WNV disease symptoms was taken into account and therefore environmental data used for this study was taken for the month of July, the month prior to peak WNV human incidence cases (Campbell et al., 2002).

\section{Least squares regression (LSR) modeling}

WNV disease annual incidence rate (cases per 100,000 populations) was used as the measure of disease severity in this study. Annual WNV-infected dead birds sentinel data, averaged for 2004-2010, was used as a surrogate of WNV risk and was the dependent variable for modeling purposes in this study because several other studies (Chaintoutis et al., 2014; Eidson et al., 2001a; Eidson et al., 2001b; Eidson et al., 2001c; Guptill et al., 2003; Johnson et al., 2006; Mostashari et al., 2003; Nielsen \& Reisen, 2007; Patnaik, Juliusson \& Vogt, 2007; Ruiz et al., 2004) have suggested links between infected dead birds and WNV human infection rates. Since wild birds are the primary reservoir hosts for WNV and indicator of human infection risk, we utilized this association to develop the disease prediction model. We determined the utility of this relationship by correlating the dead birds data with the human incidence rate $\left(r^{2}=0.409\right.$, determined by utilizing Ordinary Least Squares modeling within the Modeling Spatial Relationships tools within the Spatial Statistics Tools toolbox incorporated in ArcGIS ${ }^{\circledR}$ Arc Toolbox). While infected dead bird counts only explain about $40 \%$ of reported human cases in California, it is a highly significant predictor $(p=0.01)$. Hence we used a dead bird model, with infected dead birds as a dependent variable, to assess WNV risk among human population. 
Interpretations of ordinary Least Squares Regression (LSR) model performance were based on assessing multi-collinearity, robust probability, adjusted $R^{2}$ and Akaike's information criterion (AIC) (Akaike, 1974). Multi-collinearity was assessed through the variance inflation factor (VIF) statistic, which measures redundancy among explanatory variables. Explanatory variables associated with VIF values larger than about 7.5 indicate that these variables are providing similar information, and they were removed one at a time from the model based on VIF value until the model became unbiased. Robust probability indicates the statistically significant variables that are important to the regression model. Examining VIF values and robust probability, we ran and re-ran LSR models until narrowing down to non-redundant and significant variables: land surface temperature; stream density, and; road density. Akaike's information criterion (AIC) was then used to determine the best LSR model.

The next step was to explore GWR models that might better explain the variation in infected dead bird counts based upon environmental data. Spatial autocorrelation (Global Moran's I) was utilized to assess whether the environmental factors exhibited a random spatial pattern (Goodchild, 1986), and where adequate models have a random distribution of the residuals (Mitchell, 2005).

\section{Geographically weighted regression (GWR) modeling}

Under conditions of non-stationarity in LSR modeling, geographically weighted regression (GWR) was explored to potentially improve modeling results. These results were determined by utilizing Geographically Weighted Regression modeling within the Modeling Spatial Relationships tools within the Spatial Statistics Tools toolbox incorporated in ArcGIS ${ }^{\circledR}$ Arc Toolbox. The same explanatory variables that were used in LSR modeling were used to run GWR rather than starting with the full global set of parameters so as to avoid introducing "improvement" that could not be attributed solely to which modeling approach was applied. In other words, if GWR modeling was not applied to the same variables as LSR modeling, but yielded better results, we would not know if the improvement was due to the modeling approach or the environmental data that was used to build each model.

Once key environmental factors were identified during LSR modeling, we proceeded to explore the spatial variability of local regression coefficients to determine whether the underlying process exhibited spatial heterogeneity (Fotheringham, Brunsdon \& Charlton, 2003). A GWR local model was applied to analyze how the relationship between infected dead bird counts and environmental factors changed from one county to another. Unlike conventional LSR regression modeling, which produces a single regression equation to summarize global relationships among the independent and dependent variables, GWR detects spatial variation within relationships in a model and produces information useful for exploring and interpreting spatial non-stationarity (Fotheringham, Brunsdon \& Charlton, 2003).

A spatial kernel was used to provide geographic weighting for the local window centered on the grid nodes used in our model. There are two possible categories of spatial kernels: fixed/adaptive and bandwidth, which is a key coefficient that controls the size of the 
kernel (Akaike, 1974). These kernels tend to be Gaussian or Gaussian-like which implies that distant samples are weighed lesser than the proximal ones. There are three potential bandwidth approaches: Akaike information criterion (AIC), cross validation (CV), and bandwidth parameter. For our GWR model, the AIC approach was chosen because the distribution of infected dead birds was not consistent in the study area. The following settings were used in ArcGIS GWR: bandwidth method = AIC and Kernel type = Adaptive.

Finally, we examined independency and normality of residuals, to evaluate the fit of the model. Local collinearity, the square root of the largest eigenvalue divided by the smallest eigenvalue, of our GWR model was also assessed but no data points were removed as they compromised model diagnostics. The adjusted coefficient of determination (Adjusted $R^{2}$ ) was used for comparing LSR and GWR models to determine which approach would provide a better understanding of the relationship between environmental conditions and West Nile Virus risk (Fotheringham, Brunsdon \& Charlton, 2003).

\section{RESULTS}

LSR modeling identified land surface temperature (VIF $=1.046)$, stream density (VIF $=1.177)$, and road density $(\mathrm{VIF}=1.143)$ as statistically significant $(p<0.05)$ variables related to WNV risk:

$\mathrm{WNV}$ risk $=-75.87+595.60(R D)+1.89(L S T)-146.89(S D)$

Where:

WNV risk $=$ average infected dead bird count

$R D=$ road density

$L S T=$ land surface temperature

$S D=$ stream density.

The histogram of the LSR model's residuals approximates that of a normal curve, with a non-significant $(0.134, p>0.05$ ) Jarque-Bera statistic (Jarque \& Bera, 1980), and the Moran's I Index $Z$-score (1.23) all imply that the model is unbiased and significantly different than random.

However, the Koenker statistic $\left(0.000007^{\star}, p<0.05\right)$ confirmed non-stationarity in the LSR model indicating that there is not a consistent relationship between the explanatory variables and WNV risk across the study area. Further, the presence of mild heteroskedasticity was noted in the LSR model. We conclude that the LSR model is stable but non-stationary, suggesting that proceeding with GWR model was warranted.

The GWR model in this study was implemented using the following algorithm:

$\mathrm{WNV} \operatorname{risk}_{(\mathrm{i})}=\beta_{\mathrm{i} 0}+\beta_{(\mathrm{i} 1)} R D_{(\mathrm{i})}+\beta_{(\mathrm{i} 2)} L S T_{(\mathrm{i})}-\beta_{(\mathrm{i} 3)} S D_{(\mathrm{i})}+\varepsilon_{(\mathrm{i})}$

where $\beta$ coefficients are county (i) specific, and $R D$ is road density, LST is land surface temperature, and $S D$ is stream density.

Comparing the fit of the global LSR model (assumes homogeneity of variables across space) and local GWR model (makes no assumption of homogeneity), we found that the global LSR adjusted $R^{2}$ was $0.61\left(R^{2}\right.$ was $0.66, P<0.05$, Fig. 1$)$ with analysis run on all 58 


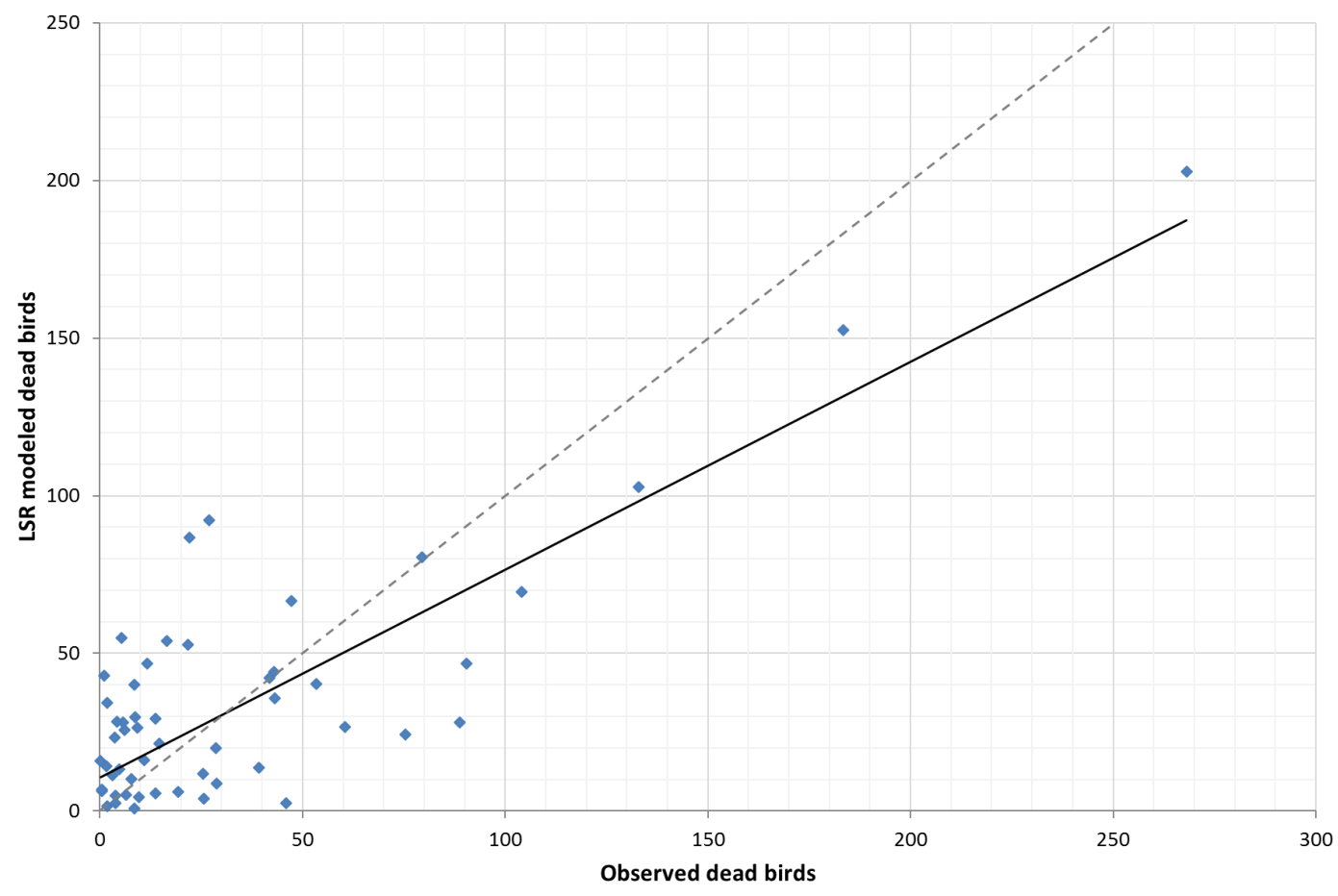

Figure 1 Trendline plot for global LSR model (model: $y=0.6591 x+10.563 ; r^{2}=0.66$ ), dashed line ideal 1:1 relationship.

counties. The local GWR adjusted $R^{2}$ was $0.71\left(R^{2}\right.$ is $0.75, p<0.05$, Fig. 2$)$ with a bandwidth of 54 , which suggests that there has been some improvement by using a local modeling approach. Our preferred measure of model fit, AIC, gave a value of 567.7 for the global model and 551.4 for the local model. The difference of 16.3 is relatively strong evidence of an improvement in the model fit to the data. Further, the problem of hetroskedasticity that was noted in the OLS model was not observed in the GWR model.

We also tested the results using different bandwidth parameter. Several iterations were run but it was observed that although a smaller band-width criterion gave an improved combination of AICc and adjusted $R^{2}$ values, it also compromised the model diagnostics by introducing local collinearity and thus instability in the model. Addressing local collinearity by removing the Counties having condition number greater than 30 affected the model's overall results. Thus, it is better to have a larger band-width rather than violating model assumptions and to avoid the unstable prediction (Charlton \& Fotheringham, no date; Nakaya, 2014).

Mapping the values of the standardized residual across California (Fig. 3A) provides a representation of: (a) areas with unusually high or low residuals and (b) whether the residuals were spatially autocorrelated. Counties with excessively large positive residuals would under-predict WNV risk, and counties with excessively large negative residuals would over-predict WNV risk. The spatial autocorrelation of GWR residuals for our model resulted in a Moran's I value of $-0.11(p=0.18)$, implying little evidence of any autocorrelation in them. 


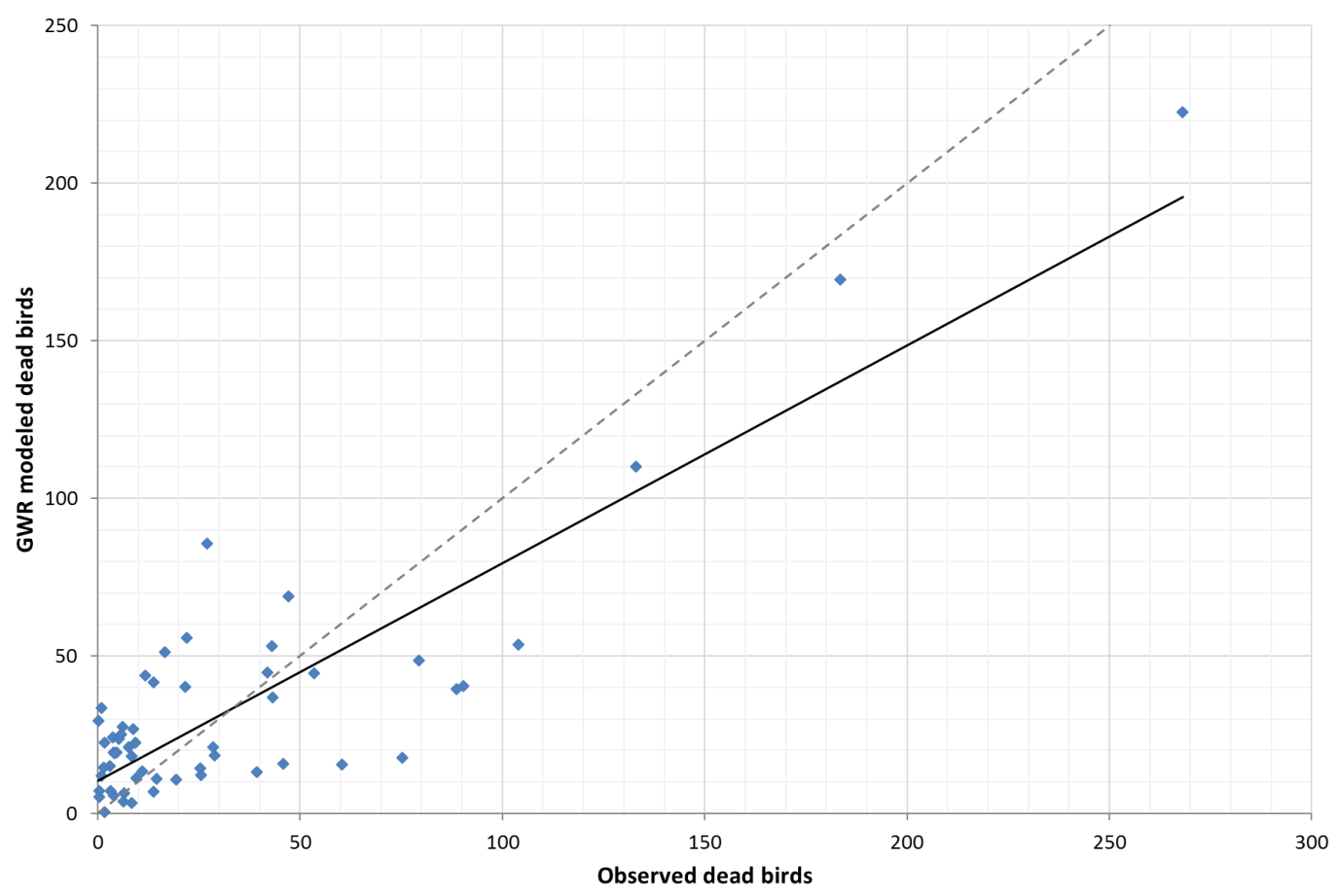

Figure 2 Trendline plot for local GWR model (model: $\left.y=0.6911 x+10.259 ; r^{2}=0.75\right)$, dashed line ideal 1:1 relationship.

Local coefficient estimates for significant factors were mapped using quantile classification method. Figure 3B shows the variation in the model's coefficient estimates for the land surface temperature (LST) variable. The map for the local coefficients reveals that the influence of this variable in the model varies considerably over California, with a strong north-south direction. The range of the local coefficient is from 1.26 for the northernmost counties to 3.06 for the southernmost counties-evidence that points to heterogeneity in the model structure within California. The global coefficient and all the local coefficients for this variable are positive-there is agreement between the two models on the direction of the influence of this variable. Figure $3 \mathrm{C}$ shows a similar distribution in north-south direction of positive road density coefficient. Figure 3D reveals the opposite for stream density coefficients, with larger values in the north and smaller values in south. Contrary to our initial thoughts, stream density demonstrated a negative relation to disease risk. This may reflect that flowing water is normally not suitable for larval development of the various species of mosquitos that commonly transmit WNV or that rasterizing the stream database into stream density introduces a component that is not yet fully understood.

Our best ordinary least squares model, the global LSR model (Eq. 1) produced an adjusted $R^{2}$ of $0.61(p<0.05)$ with a corresponding corrected AIC of 567.70. Utilizing the same environmental variables, our best local GWR model (Eq. 2) produced an adjusted $R^{2}$ of $0.71(p<0.05)$ with a corresponding corrected AIC of 551.4. A 16 point decrease in the AIC and approximately $16 \%$ improvement in the model performance suggest that incorporating spatial data improves the predictive ability of WNV risk. 

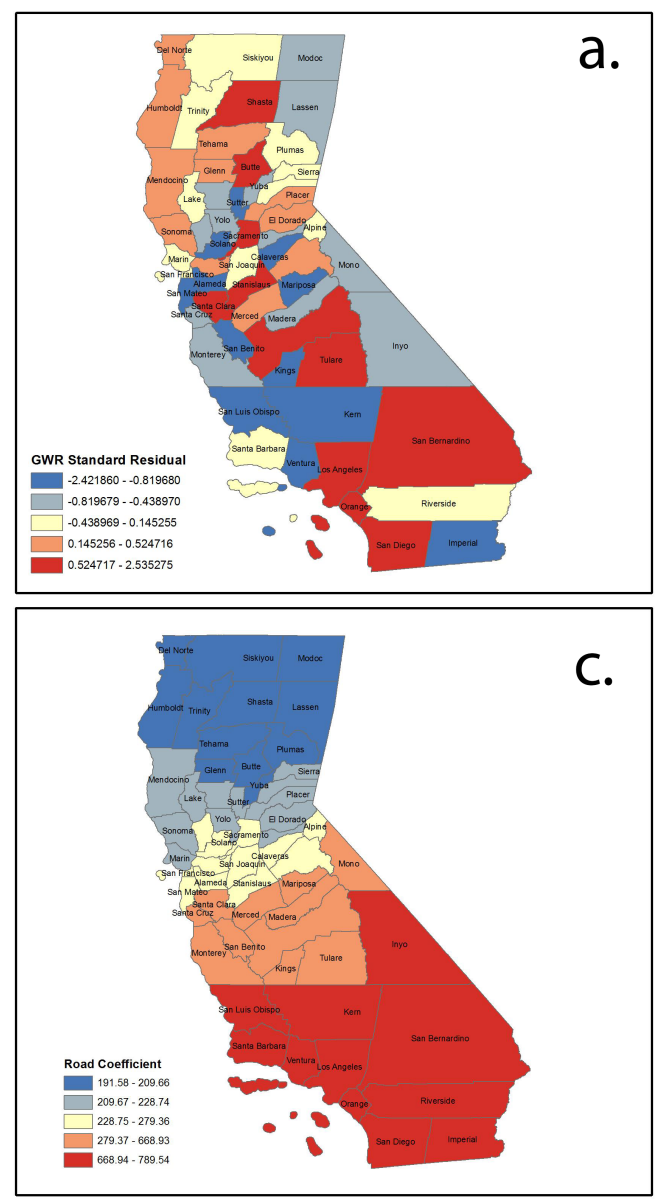
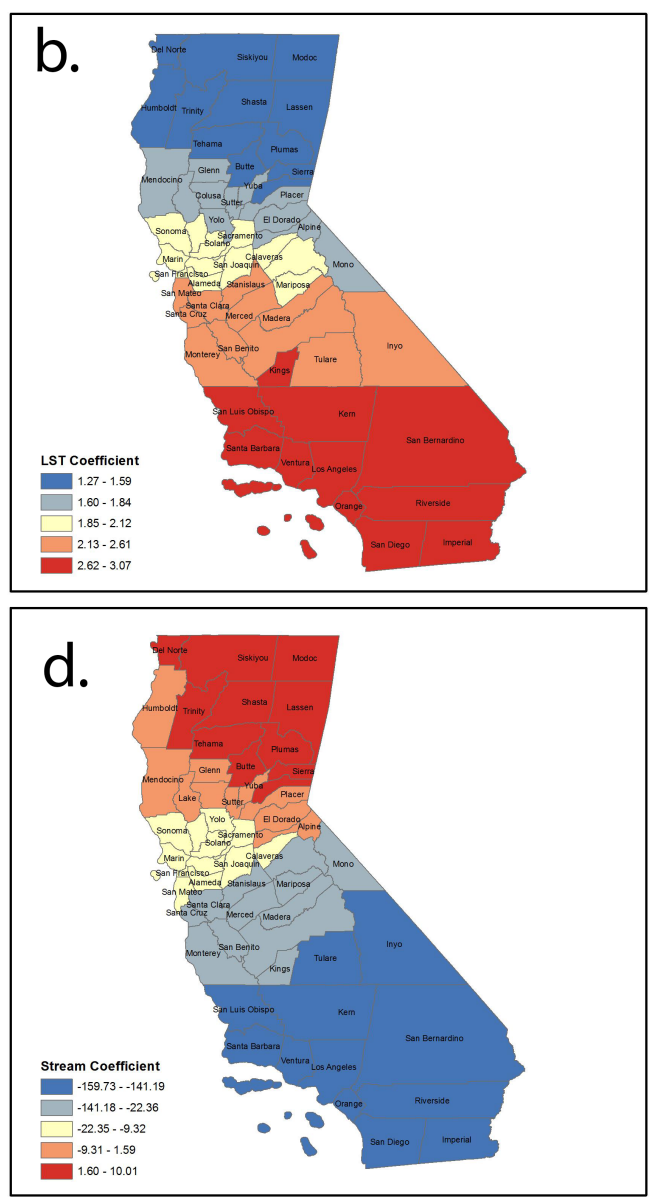

Figure 3 Spatial distribution of (A) standardized residuals; (B) land surface temperature coefficients; (C) road density coefficients; and (D) stream coefficients.

\section{CONCLUSIONS}

One of the frequent technical issues in modeling disease risk is to incorporate local rather than global associations in these models (Foley, Charlton \& Fotheringham, 2009). In spatial regression models, a global model can be used to examine the relationship between disease risk and potential explanatory factors which are based on the assumption that the relationship is a stationary spatial process (Miller, 2012). For a small and homogenous region of interest, it is reasonable to assume that the explanatory factors would not change significantly across the region, and the relationship between WNV risk and the potential factors would also be unchanged. However, important variables such as topography, climate, and population distribution change greatly when it comes to a large region like California with an area of over 163,000 square miles. California is geographically diverse and is equally varied in its range of climates with several climatic sub-regions recognized. It would be unexpected to find that the spatial stationarity assumption holds in such a large area. 
The distinct north-south pattern revealed in our study could be attributable to typical latitudinal expressions of temperature and precipitation, especially since California has a north-south length of $1,350 \mathrm{~km}$. This environmental pattern is also a likely contributor to the distribution of different mosquito species in the United Stated, especially notable in its manifestation in California. A recent report (CDC, 2013) shows that while $C x$. Tarsalis is distributed throughout California, $C x$. pipiens is a more important mosquito vector in northern California, while $C x$. quinquefasciatus is more important in southern California. While WNV can be found in a wide variety of ecosystems, the north-south pattern of infected birds detected in this study may be expressed more noticeably in California due to the north-south differences in mosquito species distributions as observed in the Centers for Disease Control report.

Our results concur that understanding WNV risks is improved when considering spatial heterogeneity of the variables that affect the risk (Beck et al., 1994). Besides improving prediction accuracy, spatial heterogeneity can also provide insights into the underlying ecological processes controlling the distributions of vector populations and zoonotic pathogens (Wimberly, Baer \& Yabsley, 2008) because GWR models consider spatial heterogeneity by separating the large heterogeneous region into smaller, more homogeneous local regions. Fotheringham (Fotheringham, 2009a), stated that an advantage of using GWR is that it accounts for much of the spatial autocorrelation in the residuals that is usually found in global modeling. Further, it is possible that a variable that is insignificant at the global level might be important locally.

There are several limitations of this study. First, it is assumed that factors suitable for mosquito habitat increase the likelihood of WNV spread in human populations. On the surface this seems to be reasonably apparent; however, we do not have specific evidence that this is true. Second, it is also assumed that the probability of human infection is higher in counties with multiple confirmed WNV bird cases, another reasonable conjecture with several references in the literature, but without direct confirmation. Potential problems with this assumption include varying human population density (e.g., two areas with the same number of infected dead birds reported but one area's human population density is substantially different than the other), variations in level of public concern (as reports of infected dead birds increase, more people begin looking for dead birds), and resource availability might bias the reporting of dead birds (wealthy areas devote disproportionate resources to the issue). Thus, proper surveillance methods that take into consideration these limitations while collecting infected dead bird data will contribute to more meaningful results. Third, our approach assumes that people are infected within the county of their residence, ignoring the possibility of contracting an infection while traveling outside the county limits. Lastly, road density could also be correlated with dead bird surveillance effort and might be a potential bias for reporting dead birds. We recognize that if these assumptions do not hold, modeling WNV risks based on infected dead birds may yield biased results. However, if the assumptions do hold, the local modeling approaches should improve predictions of WNV risks.

The research described in this paper suggests that a spatially explicit local model using GWR approaches to adjust for spatial autocorrelation and non-stationarity can yield 
improved predictions compared to ordinary LSR modeling of WNV risk. A spatially explicit modeling technique may be useful in policy-making and decision-making depending on the granularity and resolution of available data. Identifying the spatial variations in relationships by estimating local regression parameters allows the spatial distribution and interaction of predictor variables to be explored. Analyzing local variations in relationships provides those concerned with public health policy the ability to target resources and to achieve improved outcomes through location-specific activities (Comber, Brunsdon \& Radburn, 2011) because spatial heterogeneity can improve predictions by capturing geographic shifts in the ecological drivers (Wimberly, Baer \& Yabsley, 2008). While environmental data used in this research were of fine resolution, WNV disease human incidence data and infected dead bird data that is used is available only at a coarser county scale. We had to assume that aggregating the environmental data up to the county adequately represented the environmental conditions presented in the county, but we knew that data aggregation was likely to introduce some uncertainty into the model. The dead bird model applied in this study can be used for better understanding of WNV risk and the techniques used could be replicated at finer spatial scales thus leading to better intervention efforts.

In summary, WNV, a globally emerging infectious disease, was found to be heterogeneously related to environmental factors at the county level throughout California during the time that our data were collected. Our findings may assist those conducting risk assessments for WNV transmission in local areas by helping local public health entities allocate resources and improve preparedness for an outbreak according to region-specific conditions.

\section{ACKNOWLEDGEMENTS}

The authors wish to thank the Center for Computational Epidemiology and Response Analysis, the Center for Remote Sensing, and the Advanced Environmental Research Institute at the University of North Texas for technical advice and access to software and hardware to undertake this project.

\section{ADDITIONAL INFORMATION AND DECLARATIONS}

\section{Funding}

Funding was provided by the Advanced Environmental Research Institute, University of North Texas. The funders had no role in study design, data collection and analysis, decision to publish, or preparation of the manuscript.

\section{Grant Disclosures}

The following grant information was disclosed by the authors:

Advanced Environmental Research Institute.

University of North Texas.

\section{Competing Interests}

The authors declare there are no competing interests. 


\section{Author Contributions}

- Abhishek K. Kala conceived and designed the experiments, performed the experiments, analyzed the data, contributed reagents/materials/analysis tools, wrote the paper, prepared figures and/or tables, reviewed drafts of the paper.

- Chetan Tiwari, Armin R. Mikler and Samuel F. Atkinson conceived and designed the experiments, performed the experiments, analyzed the data, contributed reagents/materials/analysis tools, wrote the paper, reviewed drafts of the paper.

\section{Data Availability}

The following information was supplied regarding data availability:

Kala, Abhishek; Atkinson, Sam; Tiwari, Chetan; Mikler, Armin (2017): Data and data dictionary-WNV Modeling. figshare. https://doi.org/10.6084/m9.figshare.4596859.v1.

\section{REFERENCES}

Akaike H. 1974. A new look at the statistical model identification. Automatic Control, IEEE Transactions on 19:716-723 DOI 10.1109/TAC.1974.1100705.

Atkinson SF, Sarkar S, Aviña A, Schuermann JA, Williamson P. 2012. Modelling spatial concordance between Rocky Mountain spotted fever disease incidence and habitat probability of its vector Dermacentor variabilis (American dog tick). Geospatial Health 7:91-100 DOI 10.4081/gh.2012.108.

Atkinson SF, Sarkar S, Aviña A, Schuermann JA, Williamson P. 2014. A determination of the spatial concordance between Lyme disease incidence and habitat probability of its primary vector Ixodes scapularis (black-legged tick). Geospatial Health 9:203-212 DOI 10.4081/gh.2014.17.

Barrett AD. 2014. Economic burden of West Nile virus in the United States. The American Journal of Tropical Medicine and Hygiene 90:389-390

DOI 10.4269/ajtmh.14-0009.

Beck LR, Rodriguez MH, Dister SW, Rodriguez AD, Rejmankova E, Ulloa A, Meza RA, Roberts DR, Paris JF, Spanner MA. 1994. Remote sensing as a landscape epidemiologic tool to identify villages at high risk for malaria transmission. The American Journal of Tropical Medicine and Hygiene 51:271-280.

Blair C. 2009. Vector biology and West Nile virus. In: Diamond M, ed. West Nile Encephalitis virus infection: viral pathogenesis and the host immune response. New York: Springer, 45-61.

Brownstein JS, Rosen H, Purdy D, Miller JR, Merlino M, Mostashari F, Fish D. 2002. Spatial analysis of West Nile virus: rapid risk assessment of an introduced vectorborne zoonosis. Vector Borne and Zoonotic Diseases 2:157-164.

Campbell GL, Marfin AA, Lanciotti RS, Gubler DJ. 2002. West Nile virus. The Lancet Infectious Diseases 2:519-529 DOI 10.1016/S1473-3099(02)00368-7.

CDC. Species of dead birds in which West Nile virus has been detected, United States, 1999-2012. Available at http://www.cdc.gov/westnile/ resources/pdfs/Bird\% 20Species\%201999-2012.pdf (accessed on 15 January 2014). 
CDC. 2000. Update: West Nile virus activity—Northeastern United States, 2000. MMWR. Morbidity and Mortality Weekly Report 49:820-822.

CDC. 2013. West Nile virus in the United States: guidelines for surveillance, prevention, and control. Fort Collins: US Department of Health and Human Services, p 69.

Centers for Disease Control and Prevention (CDC). 2001. West Nile virus activityeastern United States, 2001. MMWR. Morbidity and Mortality Weekly Report 50:617-619.

Chaintoutis SC, Dovas CI, Papanastassopoulou M, Gewehr S, Danis K, Beck C, Lecollinet S, Antalis V, Kalaitzopoulou S, Panagiotopoulos T. 2014. Evaluation of a West Nile virus surveillance and early warning system in Greece, based on domestic pigeons. Comparative Immunology, Microbiology and Infectious Diseases 37:131-141 DOI 10.1016/j.cimid.2014.01.004.

Charlton M, Fotheringham A. Geographically weighted regression: a tutorial on using GWR in ArcGIS 9.3. National Centre for Geocomputation. National University of Ireland, 5-6. Available at http://www.geos.ed.ac.uk/ gisteac/fspat/gwr/gwr_arcgis/ GWR_Tutorial.pdf.

Comber AJ, Brunsdon C, Radburn R. 2011. A spatial analysis of variations in health access: linking geography, socio-economic status and access perceptions. International Journal of Health Geographics 10:44-55 DOI 10.1186/1476-072X-10-44.

Cooke WH, Grala K, Wallis RC. 2006. Avian GIS models signal human risk for West Nile virus in Mississippi. International Journal of Health Geographics 5:36-56 DOI 10.1186/1476-072X-5-36.

Curtis A, Ye X, Heob E, Targhetta J, Salvato V, Reyna M, Bueno Jr R, Holmes L. 2014. A comparison of three approaches to identify West Nile Virus mosquito spacetime hotspots in the Houston Vicinity for the period 2002-2011. Applied Geography 51:58-64.

DeGroote J, Sugumaran R, Brend S, Tucker B, Bartholomay L. 2008. Landscape, demographic, entomological, and climatic associations with human disease incidence of West Nile virus in the state of Iowa, USA. International Journal of Health Geographics 7:197-206 DOI 10.1186/1476-072X-7-19.

DeGroote JP, Sugumaran R, Ecker M. 2014. Landscape, demographic and climatic associations with human West Nile virus occurrence regionally in 2012 in the United States of America. Geospatial Health 9:153-168.

Dewan AM, Corner R, Hashizume M, Ongee ET. 2013. Typhoid fever and its association with environmental factors in the Dhaka metropolitan area of Bangladesh: a spatial and time-series approach. PLOS Neglected Tropical Diseases 7:e1998 DOI 10.1371/journal.pntd.0001998.

Eidson M, Komar N, Sorhage F, Nelson R, Talbot T, Mostashari F, McLean R, Group, West Nile Virus Avian Mortality Surveillance Group. 2001a. Crow deaths as a sentinel surveillance system for West Nile virus in the northeastern United States, 1999. Emerging Infectious Diseases 7:615-620 DOI 10.3201/eid0704.017402. 
Eidson M, Kramer L, Stone W, Hagiwara Y, Schmit K, Team NYSWNVAS. 2001b. Dead bird surveillance as an early warning system for West Nile virus. Emerging Infectious Diseases 7:631 DOI 10.3201/eid0704.017405.

Eidson M, Miller J, Kramer L, Cherry B, Hagiwara Y, Group WNVBMA. 2001c. Dead crow densities and human cases of West Nile virus, New York State, 2000. Emerging Infectious Diseases 7:662 DOI 10.3201/eid0704.017411.

Foley R, Charlton MC, Fotheringham AS. 2009. GIS in health and social care planning. In: Handbook of theoretical and quantitative geography. UNIL-FGSE-Workshop series (2). Lausanne: Univ. de Lausanne-Faculté des géosciences et de l'environnement, 73-115.

Fotheringham AS. 2009a. Geographically weighted regression. In: The Sage handbook of spatial analysis. London: Sage Publications, 243-254.

Fotheringham AS. 2009b. "The problem of spatial autocorrelation" and local spatial statistics. Geographical Analysis 41:398-403 DOI 10.1111/j.1538-4632.2009.00767.x.

Fotheringham AS, Brunsdon C, Charlton M. 2003. Geographically weighted regression: the analysis of spatially varying relationships. Chichester: John Wiley \& Sons, 27-64.

Goddard LB, Roth AE, Reisen WK, Scott TW. 2002. Vector competence of California mosquitoes for West Nile virus. Emerging Infectious Diseases 8:1385-1391 DOI 10.3201/eid0812.020536.

Goodchild MF. 1986. Spatial autocorrelation. Norwich: Geo Books.

Goovaerts P. 2008. Geostatistical analysis of health data: state-of-the-art and perspectives. In: Soares A, Pereira MJ, Dimitrakopoulos R, eds. GeoENV VI—geostatistics for environmental applications. The Netherlands: Springer-Verlag, 3-22.

Guptill SC, Julian KG, Campbell GL, Price SD, Marfin AA. 2003. Early-season avian deaths from West Nile virus as warnings of human infection. Emerging Infectious Diseases 9:483-484 DOI 10.3201/eid0904.020421.

Hayes EB, Komar N, Nasci RS, Montgomery SP, O’Leary DR, Campbell GL. 2005. Epidemiology and transmission dynamics of West Nile virus disease. Emerging Infectious Diseases 11:1167-1173 DOI 10.3201/eid1108.050289a.

Hu M, Li Z, Wang J, Jia L, Liao Y, Lai S, Guo Y, Zhao D, Yang W. 2012. Determinants of the incidence of hand, foot and mouth disease in China using geographically weighted regression models. PLOS ONE 7:e38978

DOI 10.1371/journal.pone.0038978.

Jarque CM, Bera AK. 1980. Efficient tests for normality, homoscedasticity and serial independence of regression residuals. Economics Letters 6:255-259 DOI 10.1016/0165-1765(80)90024-5.

Jean CM, Honarmand S, Louie JK, Glaser CA. 2007. Risk factors for West Nile virus neuroinvasive disease, California, 2005. Emerging Infectious Diseases 13:1918-1920 DOI 10.3201/eid1312.061265.

Johnson GD, Eidson M, Schmit K, Ellis A, Kulldorff M. 2006. Geographic prediction of human onset of West Nile virus using dead crow clusters: an evaluation of year 2002 data in New York State. American Journal of Epidemiology 163:171-180. 
Kilpatrick AM. 2011. Globalization land use and the invasion of West Nile virus. Science 334:323-327.

Kuehn BM. 2012. Record heat may have contributed to a banner year for west nile virus. JAMA 308:1846-1848.

Kupfer JA, Farris CA. 2007. Incorporating spatial non-stationarity of regression coefficients into predictive vegetation models. Landscape Ecology 22:837-852 DOI 10.1007/s10980-006-9058-2.

Legendre P. 1993. Spatial autocorrelation: trouble or new paradigm? Ecology 74:1659-1673 DOI 10.2307/1939924.

Liu H, Weng Q. 2012. Environmental factors and risk areas of West Nile Virus in southern California, 2007-2009. Environmental Modeling \& Assessment 17:441-452 DOI 10.1007/s10666-011-9304-0.

Liu Y, Jiang S, Wang R, Li X, Yuan Z, Wang L, Xue F. 2011. Spatial epidemiology and spatial ecology study of worldwide drug-resistant tuberculosis. International Journal of Health Geographics 10:50 DOI 10.1186/1476-072X-10-50.

Miller JA. 2012. Species distribution models Spatial autocorrelation and non-stationarity. Progress in Physical Geography 36:681-692 DOI 10.1177/0309133312442522.

Mitchell A. 2005. The ESRI guide to GIS analysis: spatial measurements and statistics. Vol. 2. Redlands: ESRI Press.

Mostashari F, Kulldorff M, Hartman JJ, Miller JR, Kulasekera V. 2003. Dead bird clusters as an early warning system for West Nile virus activity. Emerging Infectious Diseases 9:641-646 DOI 10.3201/eid0906.020794.

Nakaya T. 2014. GWR4 user manual. WWW document. Available at https:// geodacenterasuedu/drupal_files/gwr/GWR4manualpdf.

Nash D, Mostashari F, Fine A, Miller J, O'Leary D, Murray K, Huang A, Rosenberg A, Greenberg A, Sherman M. 2001. The outbreak of West Nile virus infection in the New York City area in 1999. New England Journal of Medicine 344:1807-1814 DOI 10.1056/NEJM200106143442401.

Nielsen CF, Reisen WK. 2007. West Nile virus-infected dead corvids increase the risk of infection in Culex mosquitoes (Diptera: Culicidae) in domestic landscapes. Journal of Medical Entomology 44:1067-1073 DOI 10.1093/jmedent/44.6.1067.

Ozdenerol E, Bialkowska-Jelinska E, Taff GN. 2008. Locating suitable habitats for West Nile Virus-infected mosquitoes through association of environmental characteristics with infected mosquito locations: a case study in Shelby County, Tennessee. International Journal of Health Geographics 7:12 DOI 10.11186/1476-072X-7-12.

Patnaik JL, Juliusson L, Vogt RL. 2007. Environmental predictors of human West Nile virus infections, Colorado. Emerging Infectious Diseases 13:1788-1790 DOI 10.3201/eid1311.070506.

Reisen W, Lothrop H, Chiles R, Madon M, Cossen C, Woods L, Husted S, Kramer V, Edman J. 2004. West Nile virus in California. Emerging Infectious Diseases 10:1369-1378. 
Rochlin I, Turbow D, Gomez F, Ninivaggi DV, Campbell SR. 2011. Predictive mapping of human risk for West Nile virus (WNV) based on environmental and socioeconomic factors. PloS One 6:e23280 DOI 10.1371/journal.pone.0023280.

Rodgers SE, Mather TN. 2006. Evaluating satellite sensor-derived indices for Lyme disease risk prediction. Journal of Medical Entomology 43:337-343

DOI 10.1093/jmedent/43.2.337.

Ruiz MO, Tedesco C, McTighe TJ, Austin C, Kitron U. 2004. Environmental and social determinants of human risk during a West Nile virus outbreak in the greater Chicago area, 2002. International Journal of Health Geographics 3:Article 8 DOI 10.1186/1476-072X-3-8.

Schurich JA, Kumar S, Eisen L, Moore CG. 2014. Modeling culex tarsalis abundance on the northern colorado front range using a landscape-level approach. Journal of the American Mosquito Control Association 30:7-20.

Srivastava A, Nagpal B, Saxena R, Subbarao S. 2001. Predictive habitat modelling for forest malaria vector species An. dirus in India-A GIS-based approach. Current Science-Bangalore 80:1129-1134.

Tobler WR. 1970. A computer movie simulating urban growth in the Detroit region. Economic Geography 46(supp1):234-240 DOI 10.2307/143141.

Trawinski P, Mackay D. 2008. Meteorologically conditioned time-series predictions of West Nile virus vector mosquitoes. Vector-Borne and Zoonotic Diseases 8:505-522.

Valiakos G, Papaspyropoulos K, Giannakopoulos A, Birtsas P, Tsiodras S, Hutchings MR, Spyrou V, Pervanidou D, Athanasiou LV, Papadopoulos N. 2014. Use of wild bird surveillance, human case data and GIS spatial analysis for predicting spatial distributions of West Nile Virus in Greece. PloS One 9:e96935 DOI 10.1371/journal.pone.0096935.

Wimberly MC, Baer AD, Yabsley MJ. 2008. Enhanced spatial models for predicting the geographic distributions of tick-borne pathogens. International Journal of Health Geographics 7:Article 15 DOI 10.1186/1476-072X-7-15.

Wimberly MC, Hildreth MB, Boyte SP, Lindquist E, Kightlinger L. 2008. Ecological niche of the 2003 west Nile virus epidemic in the northern great plains of the United States. PLOS ONE 3:e3744 DOI 10.1371/journal.pone.0003744. 